\title{
Hormone Supplying Renal Cell Sheet In Vivo Produced by Tissue Engineering Technology
}

\author{
Sachiko Sekiya, Tatsuya Shimizu, Masayuki Yamato, and Teruo Okano
}

\begin{abstract}
Regenerative medicine is a new medical field and is expected to have a profoundly positive effect in curing difficult-to-treat diseases. Cell sheet fabrication is an important tissue engineering technology used in regenerative medicine. This study investigated the creation of a hormone-releasing tissue using cell sheet technology, which could be utilized in future therapy for chronic renal disease. Renal cell sheets were fabricated on a temperatureresponsive cell culture surface with primary renal cells from adult porcine kidney. These sheets contained various kinds of renal cells that showed cyst-like formation. An important renal function is the synthesis of 1,25-dihydroxyvitamin $\mathrm{D}_{3}$, and this was confirmed in the cell sheets in vitro. Erythropoietin (EPO) production is another important renal function. This ability was also observed in the renal cell sheets in vitro, and then again after transplantation in a nude rat. In particular, the relative expression of EPO mRNA increased more under cell sheet culture conditions compared with exponential cell growth conditions. Histological analysis of the implanted renal cell sheets showed them to be Dolichos biflorus agglutinin-positive and to have regenerated renal tubular-like morphology. These results indicated that both functional and morphological regenerative renal tissues were fabricated by cell sheet technology. This study introduces a hormone-supplying treatment for renal dysfunctional diseases using engineered renal tissues. Moreover, since our renal cell sheets developed renal tubular-like structures in vivo, it holds promise for fabricating artificially engineered true renal tissue in the future.
\end{abstract}

Key words: cell sheet technology; renal tissue; tissue engineering

\section{Introduction}

A CCORDING TO REPORTS by the Japanese Society for Dialysis Therapy, the number of hemodialysis patients in Japan suffering from chronic kidney disease (CKD) exceeded 300,000 in $2010 .{ }^{1}$ CKD is defined as a functional or morphological disorder of a kidney that has continued for more than 3 months. CKD patients eventually face deteriorated renal function resulting in end-stage renal disease (ESRD), which requires hemodialysis or renal transplantation treatment. Urine production and blood purification are major renal functions, which require ESRD patients to undergo hemodialysis. However, the kidney has another important role in maintaining homeostasis in the body by secreting hormones. 1,25-Dihydroxyvitamin $\mathrm{D}_{3}$ (active vitamin $\mathrm{D}_{3}$ ) produced in the kidney works like a hormone, and its decrease in cases of renal failure results in lower absorption of calcium from the intestine, causing hypocalcemia. Moreover, defective erythropoietin (EPO) production from the kidney also causes anemia and accelerates renal failure. Patients with decreasing 1,25-dihydroxyvitamin $\mathrm{D}_{3}, \mathrm{EPO}$, and hormone secre- tion require repeated drug administration with specific blood condition monitoring. These insoluble problems with hemodialysis are severe issues for ESRD patients. Therefore, with this study we developed a new approach to hormone secretion failure in the kidney by applying cell transplantation technology.

Injection of a cell suspension is the most popular strategy for cell transplantation. However, it is often difficult to control cell destination after transplantation. How cells are delivered to a target organ or tissue affects the survival of the transplanted cells and the effectiveness of the treatment. ${ }^{2,3}$ Transplantation of a cell sheet is a better cell preparation technology to improve the viability of transplanted cells and is a highly effective treatment of disease. Cell sheet technology allows intact confluent cell harvesting as a single sheet by simple temperature reduction from a culture dish grafted with a temperature responsive polymer, without using any enzymatic treatment. ${ }^{4}$ For several diseases, various kinds of cell sheet transplantation therapies have been reported to show remarkable clinical benefits. ${ }^{5-8}$ The treatment effects not only include repair of defective morphology, but also hold

Institute of Advanced Biomedical Engineering and Science, Tokyo Women's Medical University, Tokyo, Japan. 
the possibility of supplying liquid factors such as insulin. ${ }^{9}$ Supplying kidney hormones from a cell sheet is an important treatment goal.

In this study, we tried to fabricate cell sheets that could secrete renal hormones. Therefore, cell sheets were fabricated with primary adult porcine renal cells, and we investigated 1,25-dihydroxyvitamin $\mathrm{D}_{3}$ (active vitamin $\mathrm{D}_{3}$ ) synthesis activity and EPO expression activity, both in vitro and in vivo. Moreover, the morphologic analysis of the renal cell sheets showed the potential for renal tubule regeneration by tissue engineering, which opens a door for new beneficial CKD treatments.

\section{Materials and Methods}

All procedures using animals in this study were performed in accordance with the Guidelines of Tokyo Women's Medical University on Animal Use.

\section{Primary culture of renal cells from porcine kidney}

Figure 1 illustrates the procedure utilized for cultivation of primary cells and fabrication of a renal cell sheet. Kidneys, approximately $40 \mathrm{~g}$ in weight, were collected from 6- or 7-monthold male miniature pigs. Immediately, 4-5 g of the cortex tissue was separated from the whole kidney and washed several times with Hank's balanced salt solution (without magnesium chloride and calcium chloride; Sigma-Aldrich, St. Louis, MO). Then, the renal tissue was minced and digested for $15 \mathrm{~min}$ with collagenase type II (class II; Worthington Biochemical, Lakewood, NJ) in a $37^{\circ} \mathrm{C}$ shaking water bath. The digested suspension solution, including renal cells, was collected and mixed
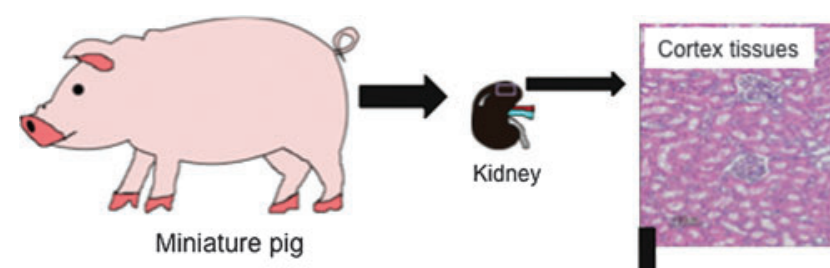

Miniature pig

Approximately $4 \times 10^{8}$ cells can be cultured from $4 \sim 5 \mathrm{~g}$ tissues

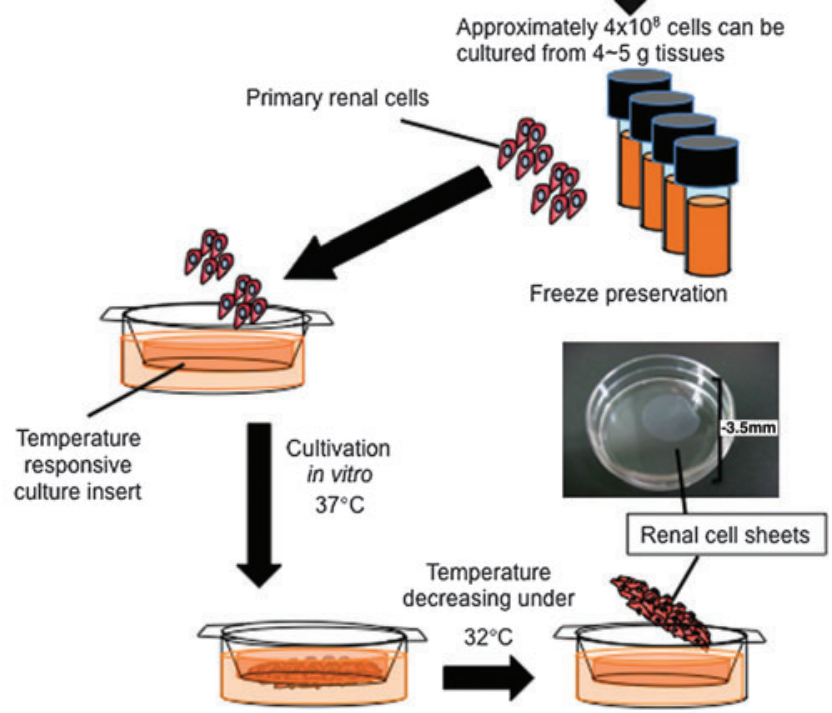

FIG. 1. Overview illustration of renal cell sheet fabrication. Schematic represents the procedure for renal cell sheet fabrication. with the same volume of Hank's buffer mixed with fetal bovine serum (FBS; Moregate Biotech, Bulimba, Australia) at $4^{\circ} \mathrm{C}$. This digesting step was repeated four times, and the cells were collected by centrifuge. After being washed with Hank's buffer and suspended with $10 \%$ FBS containing renal growth medium-2 (RGM-2; Lonza, Basel, Switzerland), the cells were seeded on dishes coated with laminin (Sigma-Aldrich) that were kept for $96 \mathrm{~h}$ at $37^{\circ} \mathrm{C}$ in a $\mathrm{CO}_{2}$ incubator. The cells that attached to the culture dishes were harvested by trypsinization, collected by centrifuge, and resuspended at approximately $6 \times 10^{6}$ cells $/ \mathrm{mL}$ with $10 \%$ dimethylsulfoxide/RGM- 2 . The re-suspended solution was dispended in $1 \mathrm{~mL}$ amounts into a 2-mL cryotube and stored in the gas phase of liquid nitrogen.

\section{Preparation renal cells}

Frozen renal cells were thawed and recultured on laminincoated culture dishes with RGM-2. Within one to two passages, renal cells were seeded on a temperature-responsive cell culture insert (UpCell ${ }^{\circledR}$, Cell Seed, Tokyo, Japan; for sixwell multiplate) at a concentration of $1 \times 10^{6}$ cells per insert. On the first day of seeding, cells were cultivated with $1 \%$ FBS of RGM2. After 24-36 h cultivation, the medium was exchanged for RGM-2. After a total of 10 days cultivation with medium changed every 1-2 days, the renal cells were harvested as renal cell sheets by reducing the culture temperature. At the time of harvesting, renal cell sheets shrink because of their cytoskeleton. The shrinking cell sheet produced a round shape with a diameter of approximately $1.5 \mathrm{~cm}$ and contained $3 \times 10^{6}$ cells $/$ sheet. In the case of samples from the exponentially growing cells, cells were seeded at a concentration of $1 \times 10^{6}$ cells for each 35-mm-diameter culture dish. After 2-3 days cultivation, confluent renal cells were harvested by a cell scraper.

\section{Histological analysis}

At appropriate periods after the transplantation or culture, the cultured or transplanted cell sheets were removed and fixed with $4 \%$ paraformaldehyde in phosphate-buffered saline (PBS). Tissue specimens were then embedded in paraffin and sliced into $5-\mu \mathrm{m}$-thick sections. The sections were stained with hematoxylin and eosin (HE) or Azan by conventional methods.

For lectin staining, the tissue samples were fixed with $4 \%$ paraformaldehyde for $1 \mathrm{~h}$ and $30 \%$ sucrose/PBS buffer for $3-5 \mathrm{~h}$ at $4^{\circ} \mathrm{C}$. Then, the samples were embedded in OTC compound and stored at $-80^{\circ} \mathrm{C}$. Cryostat sections $20 \mu \mathrm{m}$ thick were cut by a cryostat (Thermo Scientific, Walldorf, Germany). After being mounted on a silane-pretreated slide, the sections were rinsed with PBS and then incubated with Dolichos biflorus agglutinin (DBA; Vector Laboratory, Burlingame, CA) or peanut agglutinin (PNA) lectin (J-Oil Mills, Tokyo) conjugated fluorescein isothiocyanate (FITC) for $30 \mathrm{~min}$ at room temperature. The sections were counterstained with Hoechst 33342 (Wako Pure Chemicals, Tokyo) for $5 \mathrm{~min}$ to visualize cell nuclei. These thick slices were observed with a confocal microscope (LSM 510, Carl Zeiss, Göttingen, Germany).

\section{Scanning electron microscopy analysis}

Renal cell sheets were washed with ice cold $0.1 \mathrm{~mol} / \mathrm{L}$ sodium phosphate buffer and fixed with $2 \%$ glutaraldehyde 
in $0.1 \mathrm{~mol} / \mathrm{L}$ sodium phosphate buffer for $2 \mathrm{~h}$ at $4^{\circ} \mathrm{C}$. Then, samples were washed with ice cold $0.1 \mathrm{~mol} / \mathrm{L}$ sodium phosphate buffer, which was gradually replaced with a series of gradient ethanol and $0.1 \mathrm{~mol} / \mathrm{L}$ sodium phosphate buffer mixtures to dehydrate the specimens. Ethanol was then gradually replaced with $100 \% \mathrm{t}$-butyl alcohol at room temperature. The samples were dried at $-30^{\circ} \mathrm{C}$, and observed with a scanning electron microscope (VE-9800, KEYENCE, Osaka, Japan).

\section{Transmission electron microscopy analysis}

Renal cell sheets were doubly fixed with a mixture of $2 \%$ paraformaldehyde, $2 \%$ glutaraldehyde, $0.1 \mathrm{~mol} / \mathrm{L}$ sodium phosphate buffer, and $2 \%$ osmium tetroxide $\left(\mathrm{OsO}_{4}\right)$ in sodium phosphate buffer and dehydrated in a grade series of ethanol. Samples were then embedded in epoxy resin. Ultrathin sections (70 nm in thickness) were observed with a transmission electron microscope (JEOL JEM1200EX at $80 \mathrm{kV}$ ). All procedures were performed at Tokai Electron Microscopy Inc. (Aichi, Japan).

\section{$P C R$ and real-time $P C R$}

Exponentially growing renal cells, renal cell sheets, and renal tissue from miniature pigs were collected and frozen immediately at $-80^{\circ} \mathrm{C}$. Renal tissue was collected from the cortex of the kidney. Total RNA from these samples was isolated with an RNeasy Mini Kit, according to the manufacturer's protocol (Qiagen, Chatsworth, CA).

Complementary DNA was synthesized from $15 \mu \mathrm{g}$ of a RNA using a High-Capacity cDNA Reverse Transcription Kit (Invitrogen, Carlsbad, CA). Real-time PCR was performed with the TaqMan Universal PCR Master Mix (Invitrogen) and Taqman Gene Expression assay (Invitrogen), porcine Wilms' tumor 1 (WT1), EPO, cytochrome P450, family 27, subfamily B, polypeptide 1 (CYP27B1), Aquaporin-1 (AQP1), and $\beta$-actin (ACTB). PCR was performed on the generated cDNAs for megalin-1, ACTB using Ex Taq DNA polymerase (TAKARA BIO Inc, Shiga, Japan); and 40 cycles were performed. The primer pair was as follows: porcine megalin-1 forward CTG CTC TTG TAG ACC TGG GTTC, reverse TCG GCA CAG GTA CAC TCA TAAC with an expected product size of $137 \mathrm{bp}$; porcine actin- $\beta$ forward CCA GAT CAT GTT CGA GAC CTTC, reverse TCT TCA TGA GGT AGT CGG TCAG with an expected product size of $221 \mathrm{bp}$. All PCR products were purified, and DNA length was confirmed by agar electrophoresis.

\section{Measurement of 1,25-dihydroxyvitamin $D_{3}$ synthesis in vitro}

Renal cell sheets attached on a culture insert were incubated with serum free M199 medium, with or without 25hydroxyvitamin $\mathrm{D}_{3}(100 \mathrm{nmol} / \mathrm{L}$, Wako $)$ for $24 \mathrm{~h}$. To examine the effect of parathyroid hormone (PTH), human recombinant PTH (Bachem, Torrance, CA; $1 \mu \mathrm{g} / \mathrm{mL}$ ) was added to the medium. After 24-h incubation, culture medium was collected, and the production of 1,25-dihydroxyvitamin $\mathrm{D}_{3}$ was determined by an ELISA kit (Immunndiagnostik, Benshim, Germany).

\section{Transplantation of renal cell sheets onto dorsal subcutaneous tissue}

Before being detached from the temperature-responsive culture inserts, renal cell sheets were stained with CellTracker chloromethylbenzamido (CM)-DiI (Invitrogen). After being detached by lowering the culture temperature, three renal cell sheets were washed with media. Before transplantation of the cell sheets, nude rats (4 weeks old) were administrated $8 \mathrm{mg} / \mathrm{kg}$ cyclosporin (Neoral; Novartis Pharma, Basel) and anesthetized with isoflurane. An L-shaped incision $(\sim 3 \mathrm{~cm} \times$ $2 \mathrm{~cm}$ ) was made in the dorsal skin, and by lifting the incised skin, the underlying tissue was exposed. Then, three separate cell sheets were transplanted onto three separate areas of exposed tissue. The transplants were covered with a $0.5-\mathrm{mm}-$ thick silicone membrane (Unique Medical, Tokyo) to prevent the cell sheets from adhering to the dermal tissue, and the incision was closed with 5-0 nylon sutures.

\section{Porcine EPO concentration determination in blood}

Porcine EPO production in the plasma of the host rat or cultured medium was measured by ELISA (CUSABIO BIOTECH, Wuhan, China).

\section{Statistical analysis}

Results were given as mean \pm SD. Statistical analysis of the data was performed with $t$-test or analysis of variance followed by Tukey's post hoc test. Probabilities $(p<0.1,0.05$, 0.01) were considered significant.

\section{Results}

\section{Fabrication of renal cell sheets from the kidneys of adult miniature pigs}

Renal cell sheets clearly showed a multilayer cell structure (Fig. 2A, B). To compare the structures of the apical and basal sides of the renal cell sheets, they were analyzed with scanning electron microscopy. The results showed intracellular cavities created by multi-cell bridging on the apical side of the renal cell sheets (Fig. 1E). The basal side of the renal cell sheets showed a solid continuous cell sheet and smooth structures, in contrast to the apical side structure (Fig. 1F). Histological analysis showed that the tissue cross section had cyst-like formations, and Azan staining showed that the extracellular matrix was rich in the cell-cell interaction (Fig. 1B), indicating that by co-cultivating various kinds of renal cells it produced a three-dimensional (3D) structure in the renal cell sheets that resembled a native organ structure.

We also examined $\mathrm{Na}^{+} / \mathrm{K}^{+}$ATPase and laminin expressions at the lumen-like formation with immunostaining. $\mathrm{Na}^{+} / \mathrm{K}^{+}$ATPase and laminin tended to be observed at the basal side of cells around several lumen-like formations, but the polarity of cells in the renal cell sheet was immature (Fig. 2C, D). To determine the variety of cells, gene expression in the renal cell sheets was investigated by RT-PCR and compared to in vitro exponentially growing renal cells or in vivo renal tissue. WT1 and EPO are known as specific expression proteins for podocyte and EPO-expressing cells, respectively. ${ }^{10,11}$ Aquaporin-1 (AQP1) and megalin-1 are known to be highly expressed in renal tubular epithelial cells. ${ }^{12}$ The results indicated that the renal cell sheets involved podocytes, 
A

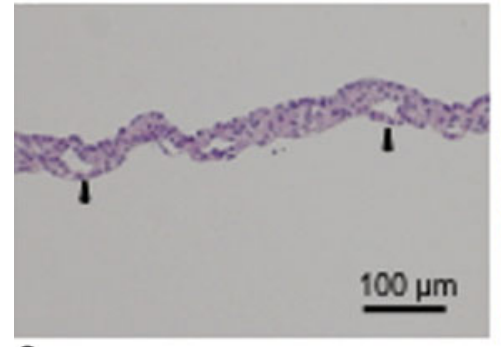

C

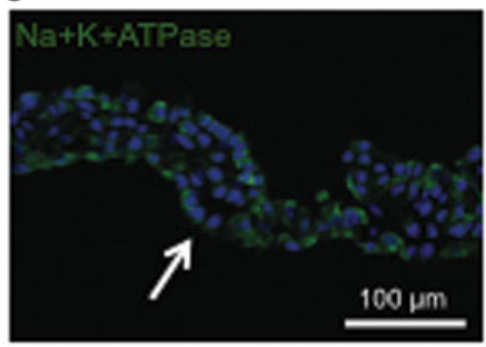

E

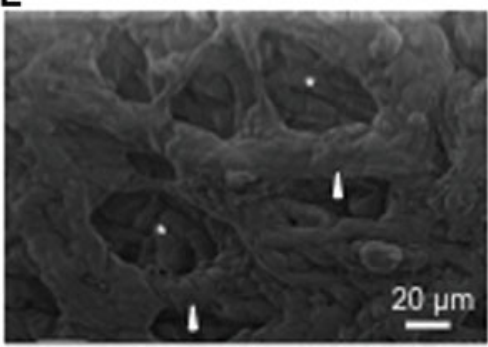

B
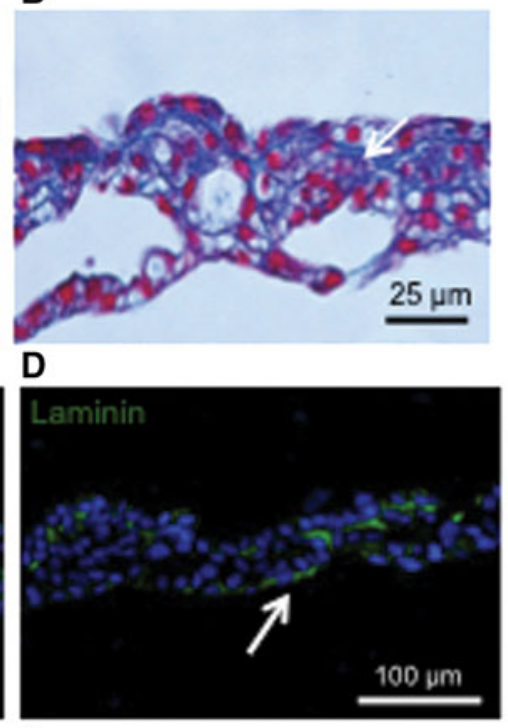

$\mathbf{F}$
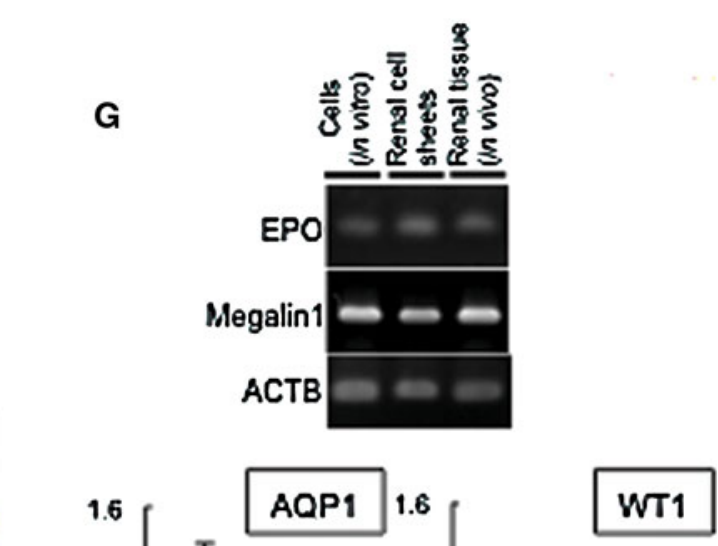

FIG. 2. The morphology of renal cell sheets. (A, B) Sections of the paraffin-embedded renal cell sheet. Specimens were stained with hematoxylin and eosin (HE) and Azan staining, respectively. Arrowheads indicate cavities or lumen formation within the renal cell sheets. (C) $\mathrm{Na}^{+} \mathrm{K}^{+}$ATPase (green) and (D) laminin (green) in renal cell sheet with immunostaining. White arrows indicate cavities or lumen formation within the renal cell sheets. (E, F) Scanning electron microscopy analysis images showing the apical (E) and basal (F) sides of the renal cell sheet. White asterisks indicate the cavities and white arrowheads indicate cells surrounding the cavities. (G) An RT-PCR representative photograph. Total RNA extracted from porcine renal tissue and primary porcine renal cells (exponential growth condition) and renal cell sheets were analyzed for EPO and Megalin-1 expression by RT-PCR. Graphs show AQP1 and WT-1 expression level of renal cell sheet relative to expression level of exponential cell growth condition by quantitative RT-PCR. EPO, erythropoietin; RT-PCR, reverse transcription-polymerase chain reaction.

EPO-expression cells, and renal tubular cells (Fig. 1G). Compared to an exponential growth cell group, the mRNA expression of AQP1 and WT1 were approximately half the level in the renal cell sheet. The pattern of gene expression indicated the fraction of podocytes and proximal renal tubular epithelial cells in renal cell sheets, or whole transplantation activity of cells. The gene expression data also showed that the key renal cell types were retained through the cell preparation process from the native renal tissue to the exponential cell culture, and the fabrication of cell sheets.

\section{Various renal tubular epithelial cells were found in renal cell sheets}

Transmission electron microscopy (TEM) identified the ultrastructures of epithelial cells in the cell sheets, which showed similarity with native tubular epithelial cell morphology. In our study, microvilli were found at the surface of the renal cell sheets in contact with the culture medium solution (Fig. 3A), and the surfaces of the inner cavities also showed microvilli (Fig. 3B). However, the cell-to-cell connections of the cells on the surface of the sheet (the white bracket in Fig. 3A) were found to be different from those cells that made up the inner cavities (the brackets in Fig. 3C). The cell-to-cell connection morphology surrounding the inner cavities was similar to typical epithelial cell-to-cell connections found in loops of Henle or collecting ducts in vivo. The electron microscopy results suggested that cells with microvilli in the renal cell sheet had the same characteristics as epithelial cells in various positions of a renal tube.

\section{1,25-dihydroxyvitamin $D_{3}$ synthesis activity in renal cell sheets in vitro}

An important kidney function is the synthesis of 1,25-dihydroxyvitamin $\mathrm{D}_{3}\left[1,25-(\mathrm{OH})_{2}\right.$ vitamin $\mathrm{D}_{3}$, calcitriol] in renal tubes from 25-hydroxyvitamin $\mathrm{D}_{3}$ by the cytochrome P450, family 27, subfamily B, polypeptide 1 (CYP27B1) enzyme. The CYP27B1 gene expression was initially examined using RT-PCR analysis. The level of expression of CYP27B1 mRNA was approximately half of the exponential growth status (Fig. 4A). To confirm the actual activity of 1,25- 
FIG. 3. Transmission electron microscopy of various renal tubular epithelial cells in renal cell sheets. (A) Cells with microvilli on the apical side of the renal cell sheet. White bracket indicates the cell-to-cell interaction of the apical-side cells. (B) Cells with microvilli inside the cavity in the renal cell sheets. (C) Representative cell-to-cell connections surrounding the cavity.
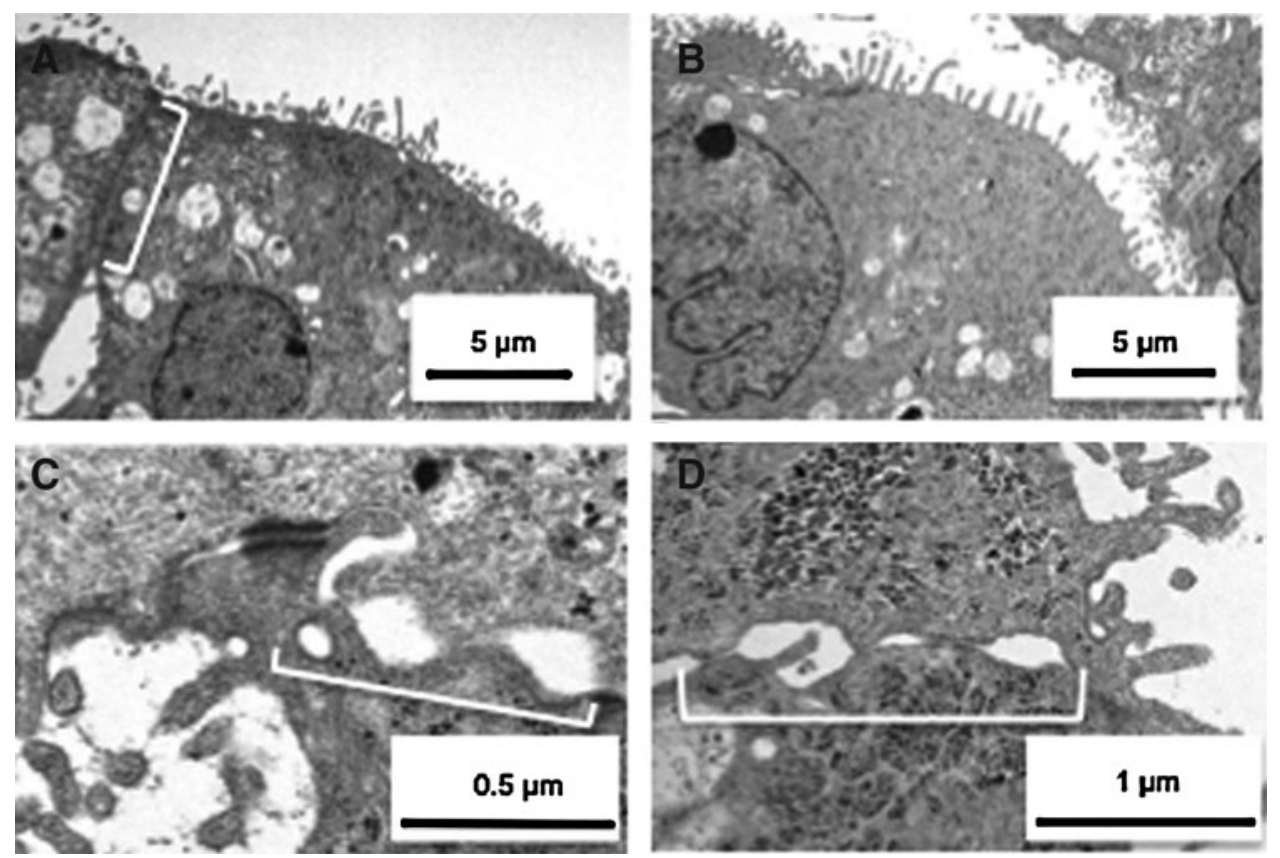

dihydroxyvitamin $\mathrm{D}_{3}$ synthesis in vitro, renal cell sheets were incubated with the precursor, 25-hydroxyvitamin $\mathrm{D}_{3}$, and this showed that 1,25-dihydroxyvitamin $\mathrm{D}_{3}$ was in fact synthesized in the renal cell sheets in vitro (Fig. 3B). Compared to exponentially growing cells, at some times the level of 1,25dihydroxyvitamin $\mathrm{D}_{3}$ synthesis was similar, but the variation in the value was much smaller in the renal cell sheet. Moreover, PTH accelerates 1,25-dihydroxyvitamin $\mathrm{D}_{3}$ synthesis in renal tubes, and also enhanced synthesis in the renal cell sheets during in vitro incubation. These data suggested that the renal cell sheet was able to perform a kidney function.

A

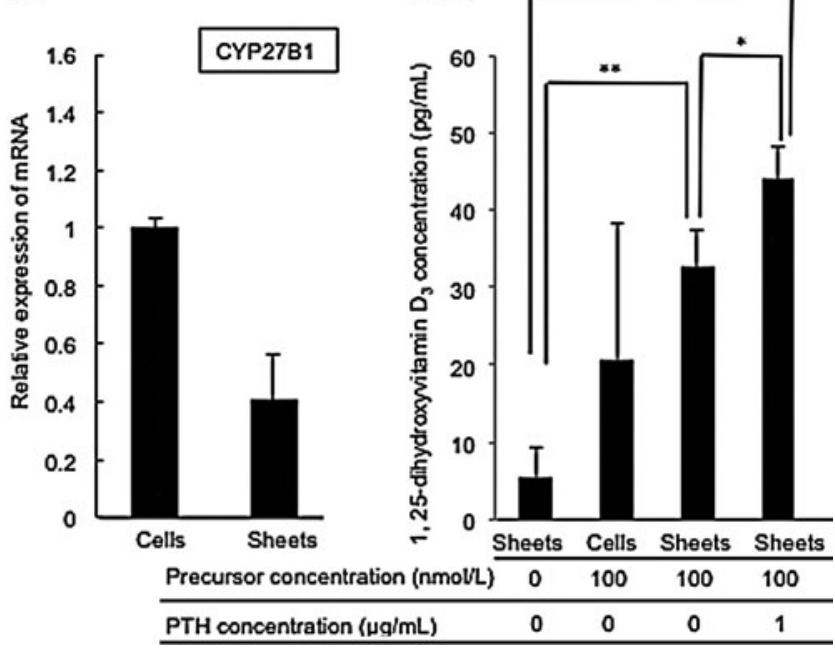

FIG. 4. The ability to synthesize 1,25-hydroxyvitamin $\mathrm{D}_{3}$ in renal cell sheets in vitro. (A) The graph shows relative expression of CYP27B1 mRNA in the renal cell sheet. (B) A graph of 1,25-dihydroxyvitamin $\mathrm{D}_{3}$ concentration in the renal cell sheet and exponential cell growth condition cultured medium $24 \mathrm{~h}$ after the addition of precursor (25-hydroxy vitamin $\mathrm{D}_{3}$ ) and parathyroid hormone (PTH). Statistically significant differences are indicated $\left({ }^{*} p<0.1,{ }^{* *} p<0.05,{ }^{* * *} p<0.01\right)$.

\section{EPO producing activity within renal cell sheets in vivo}

To examine EPO production in the renal cell sheet, EPO gene expression was analyzed. Quantitative RT-PCR results indicated that EPO gene expression increased about 30-fold in the cell sheet culture condition, compared to the exponential growth culture conditions (Fig. 5A). The EPO expression increased immediately in the cell sheet culture condition. Although the EPO expression in the renal cell sheet temporarily decreased at 5 days of cultivation, it increased again at 10 days of cultivation to return to the previous level. Next, the EPO-producing activity of the cell sheets was investigated after subcutaneous transplantation into a nude rat. The transplanted renal cell sheets prestained with CM-DiI were observed in a histological image (Fig. 5B). Since porcine renal cell sheets were found to survive in the nude rat for 7 days after the xenotransplantation, porcine EPO production in the host rat blood was investigated. Porcine EPO production was observed in the host rat blood for 3 days after transplantation. However, the production was found to decrease between day 3 and day 7 after transplantation (Fig. 5C). These results confirmed that renal cell sheets were able to produce EPO in vivo, and that transplanted renal cell sheets were able to communicate with the host blood.

\section{In vivo morphology of transplanted renal cell sheets}

After being transplanted in nude rats, the renal cell sheets showed EPO-producing ability, and the altered morphology of the cell sheets was also examined by histological analysis. DBA and PNA are known as specific lectins for renal tubes, and the ureteric buds and collecting ducts were positive for DBA lectin. PNA is also known as a renal tube-specific lectin and can also stain distal tube cells, glomerular epithelial cells, smaller collecting duct cells, and the basal membrane in pig. ${ }^{13}$ Transplanted renal cell sheets were stained with DBA conjugated with FITC or PNA conjugated with FITC. DBA-positive lumens were clearly observed 3 days after transplantation (Fig. 6B), and PNA-positive lumens were also found 3 days 


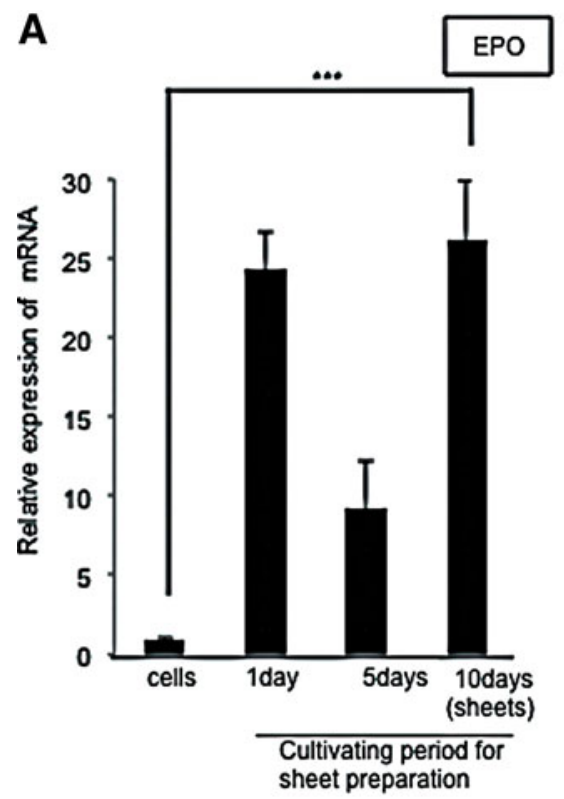

B
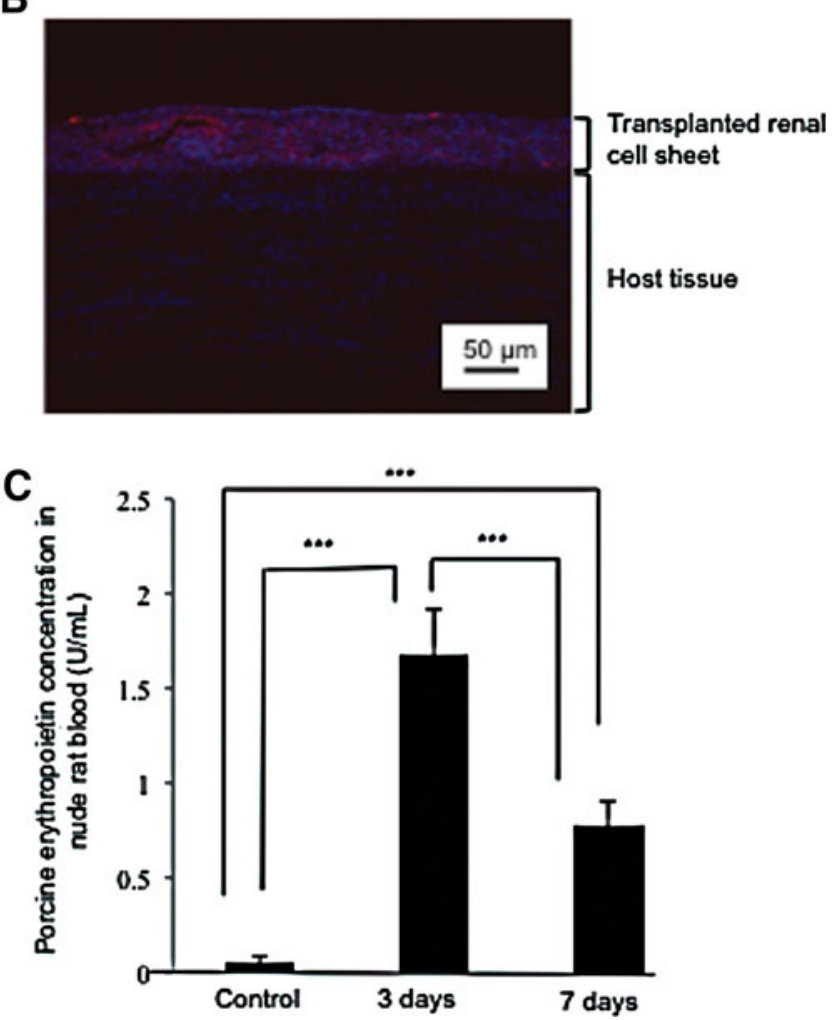

FIG. 5. The ability to produce EPO in renal cell sheets in vivo and in vitro. (A) A graph of EPO gene relative expression in primary porcine renal cells and renal cell sheets. The EPO gene relative expression level in the renal cell sheet to exponential cell growth condition. (B) A histological image of a renal cell sheet three days after transplantation. Red and blue fluorescence indicates the prestaining renal cell sheets by CM-DiI and total cell nuclei of transplanted renal cell sheet and host tissue, respectively. (C) A graph of porcine EPO concentration in host rat blood. Control indicates the basal concentration in no renal cell sheet transplanted nude rat blood. Statistically significant differences are indicated $(* * *<0.01)$. after transplantation (Fig. 6E). However, the fraction of DBApositive cells was higher than PNA-positive cells in the renal cell sheet 3 days after transplantation. Compared to renal cell sheets before transplantation, the PNA-positive cells decreased in the transplanted renal cell sheets (Fig. 6A, D). On the other hand, DBA-positive cells were positioned in the center of the renal cell sheets after transplantation. Moreover, DBA-positive morphology became more complex, and increasingly complicated tubes appeared 7 days after transplantation (Fig. 6C). Additionally, some DBA-positive budding tubular structures were observed (Fig. 6F). The results indicated that in vivo conditions were fit for growing and organizing DBA-positive cells in the renal cell sheets after transplantation. These results showed that a renal tube-like structure was partially reorganized within the renal cell sheets after transplantation.

\section{Discussion}

The renal cell sheets we obtained were found to synthesize 1,25-dihydroxyvitamin $\mathrm{D}_{3}$ in vitro and produce EPO in vitro and in vivo, suggesting the possibility of a new cell-based therapy.

Normal EPO concentration in serum is $10-30 \mathrm{mU} / \mathrm{mL}$ and the half-life time of EPO in serum is $5-9 \mathrm{~h} .{ }^{14}$ In the body, EPO transcription is activated by the onset of hypoxia, associated with hypoxia inducible factor (HIF)-1 transcription activity. ${ }^{15}$ In our study, cell sheets were created under high-density cell conditions. Because the oxygen tension near individual culture cells was estimated to be lower under cell sheet, EPO gene expression under cell-sheet conditions was higher compared to the exponentially growing cell culture conditions. It is possible to develop a clinical application to supply hormones with cell sheets. The issue for renal cell sheets was the low production level of EPO for treatment of renal anemia. If the EPO level from renal cell sheets can be maintained at 3 days after transplantation in anemic conditions, approximately 5000-fold cell sheets would be needed for and effective therapy. Thus, the renal cell sheets need to be enhanced to produce a higher EPO supply. The transfection of hEPO gene with native transcription mechanism of EPO or increasing the concentration of EPO-producing cells in the renal cell sheets need to overcome the problem in the future.

Moreover 1,25-dihydroxyvitamin $\mathrm{D}_{3}$ synthesized by CYP27B1 is positively regulated by $\mathrm{PTH} .{ }^{16,17}$ In our in vitro experiment, the renal cell sheet reacted to $\mathrm{PTH}$, suggesting that the renal cell sheet has an intact CYP27B1-activated mechanism just like in vivo. Thus, our renal cell sheet has the potential not only for EPO production, but also 1,25dihydroxyvitamin $\mathrm{D}_{3}$ production after transplantation.

To avoid any immune rejection, autologous renal cells are better potential candidates as a cell source. However, cell viability in a dysfunctional kidney will still be an obstacle. The primary culture efficiencies of renal cells are severely decreased in CKD patients. ${ }^{18}$ Although the adult kidney has the potential for regeneration, the potential of those cells is decreased in the renal failure model. ${ }^{19}$ On the other hand, induced pluripotent stem (iPS) cells are also a candidate as a cell source. ${ }^{20}$ The low induction efficiency from somatic cells is one issue, and there are variations in the efficiency of iPS cell lines for inducing target cells. Thus, controlling iPS cell differentiation will be a breakthrough for cell-based CKD therapy. 

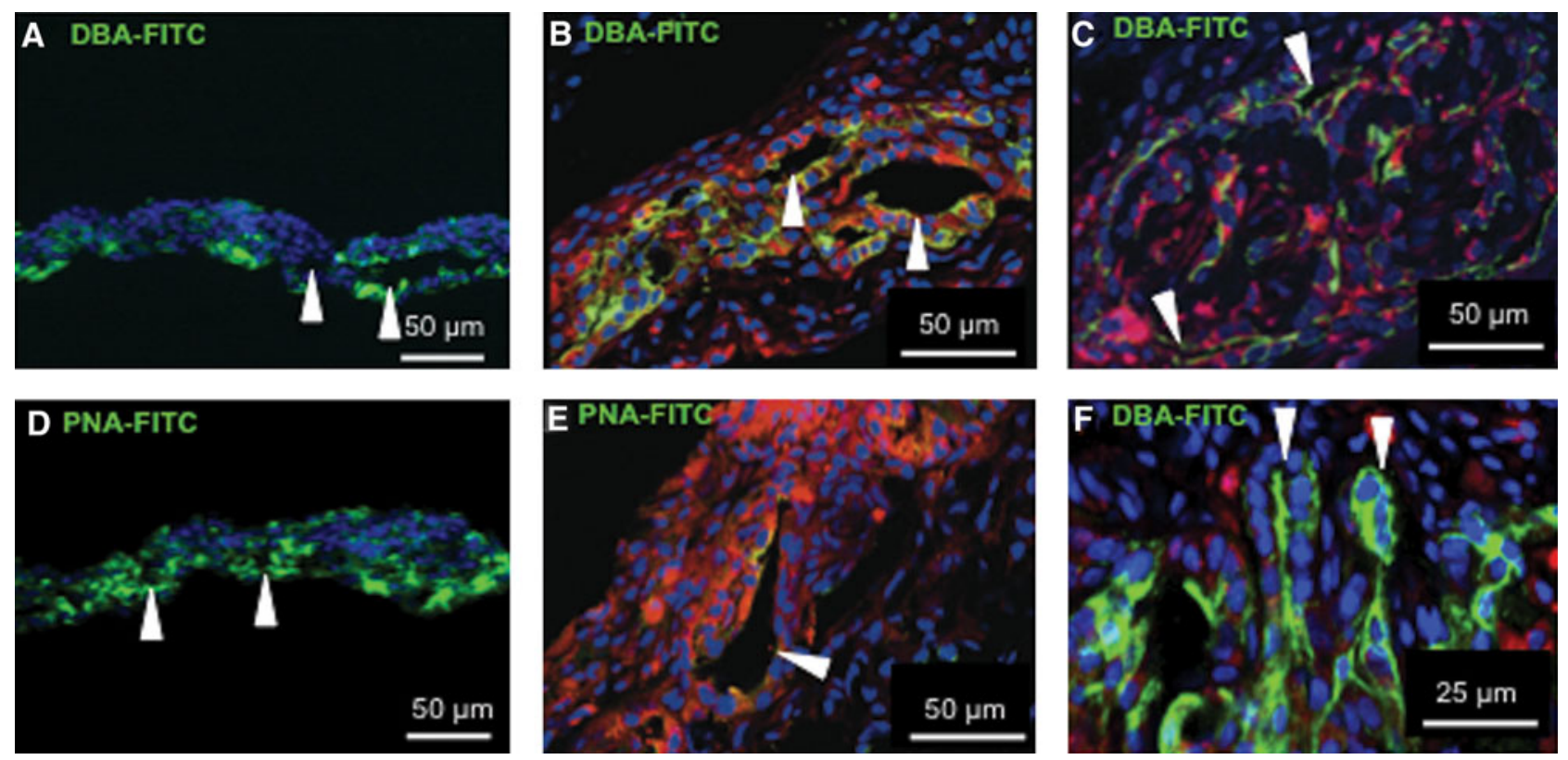

FIG. 6. Tubular reorganization in renal cell sheets after the transplantation, showing the histological analysis of renal cell sheets and transplanted renal cell sheets. In vitro renal cell sheets (A, D), and 3 days (B, E), 7 days (C, F) after transplantation are depicted. (A-C, F) show DBA-FITC staining, and (D, E) show PNA-FITC staining. Arrowheads in (A, B, D, E) indicate a lumen-like morphology in the renal cell sheets. The arrowheads in (C) indicate a tubular cross section at 7 days. The arrowheads in (F) indicate a budding structure of tubule cross section at 7 days. FITC, fluorescein isothiocyanate; PNA, peanut agglutinin.

According to other reports, in 3D collagen culture, glomeruli-like morphology regenerates with primary cells isolated from adult kidney. ${ }^{21}$ Even though primary renal adult cells may have sufficient potential for regeneration, the current in vitro culture technology cannot support their apparent ability for regeneration. One target area to produce more suitable conditions is 3D cell culture. ${ }^{22,23}$ However, scaffold utilization often hinders cell-to-cell interaction. Another candidate is layering two-dimensional cell sheets for thicker tissue fabrication. ${ }^{23,24}$ Gradually increasing the tissue thickness by layering cell sheets can solve the problem of diffusion limitation of nutrients. Some combination of these procedures may be the breakthrough for engineered renal tissue.

To fabricate urine-drainable renal tissues, ${ }^{25,26}$ blood vessels running parallel to renal tubules that can communicate solution with each other must be introduced in our renal sheet. The induction of blood vessels has been well researched, ${ }^{27-30}$ and we also need to introduce vessels within renal cell sheets. Just as with blood, urine must also be extracted from engineered renal tissues. In our result, tube formations of cells from adult kidney were also observed after transplantation. Thus, in vivo condition at the time of tube formation is an important environmental factor for ureter induction for urine extraction.

In this study, renal tissue regeneration with cell sheet technology was investigated, and the results suggested the possibility of a renal cell treatment for CKD patients. Although this study was only a preliminary trial, it holds promise for the next generation of CKD therapy with tissue engineering technology.

\section{Acknowledgments}

The present work was supported by grants for the Funding Program for World-Leading Innovative R\&D on Science and Technology (FIRST Program) from the Cabinet Office, Government of Japan (to T.O.), and Global COE (Centers of Excellence) Program by Japan's Ministry of Education, Culture, Sports, Science and Technology (MEXT).

\section{Author Disclosure Statement}

T.S. and M.Y. are consultants for CellSeed, Inc. T.O. is an Advisory Board Member for CellSeed, Inc., and an inventor/developer designated on the patent for temperatureresponsive culture surfaces. S.S. reports no competing financial interests.

\section{References}

1. Nakai S, Iseki $\mathrm{K}$, Itami $\mathrm{N}$, et al. Overview of regular dialysis treatment in Japan (as of 31 December 2010). J Jpn Soc Dialysis Ther. 2012;45:1-47.

2. Sekine H, Shimizu T, Dobashi I, et al. Cardiac cell sheet transplantation improves damaged heart function via superior cell survival in comparison with dissociated cell injection. Tissue Eng Part A. 2011;17:2973-2980.

3. Kondoh H, Sawa Y, Miyagawa S, et al. Longer preservation of cardiac performance by sheet-shaped myoblast implantation in dilated cardiomyopathic hamsters. Cardiovasc Res. 2006;69:466-475.

4. Yamato M, Utsumi M, Kushida A, et al. Thermo-responsive culture dishes allow the intact harvest of multilayered 
keratinocyte sheets without dispase by reducing temperature. Tissue Eng. 2001;7:473-480.

5. Shimizu T, Yamato M, Isoi Y, et al. Fabrication of pulsatile cardiac tissue grafts using a novel 3-dimensional cell sheet manipulation technique and temperature-responsive cell culture surfaces. Circ Res. 2002;90:e40.

6. Nishida K, Yamato M, Hayashida Y, et al. Corneal reconstruction with tissue-engineered cell sheets composed of autologous oral mucosal epithelium. N Engl J Med. 2004;351: 1187-1196.

7. Ohki T, Yamato M, Murakami D, et al. Treatment of oesophageal ulcerations using endoscopic transplantation of tissueengineered autologous oral mucosal epithelial cell sheets in a canine model. Gut. 2006;55:1704-1710.

8. Iwata T, Yamato M, Tsuchioka H, et al. Periodontal regeneration with multi-layered periodontal ligament-derived cell sheets in a canine model. Biomaterials. 2009;30:2716-2723.

9. Ohashi K, Mukobata S, Utoh R, et al. Production of islet cell sheets using cryopreserved islet cells. Transplant Proc. 2011;43:3188-3191.

10. Morrison AA, Viney RL, Saleem MA, et al. New insights into the function of the Wilms tumor suppressor gene WT1 in podocytes. Am J Physiol Renal Physiol. 2008;295:F12-17.

11. Aboushwareb T, Egydio F, Straker L, et al. Erythropoietin producing cells for potential cell therapy. World J Urol. 2008;26:295-300.

12. Baer PC, Bereiter-Hahn J, Schubert R, et al. Differentiation status of human renal proximal and distal tubular epithelial cells in vitro: Differential expression of characteristic markers. Cells Tissues Organs. 2006;184:16-22.

13. Kirkeby S, Mikkelsen HB. Distribution of the alphaGal- and the non-alphaGal T-antigens in the pig kidney: potential targets for rejection in pig-to-man xenotransplantation. Immunol Cell Biol. 2008;86:363-371.

14. Eckardt Ku, Kurtz A. Regulation of erythropoietin production. Eur J Clin Invest. 2005;35 Suppl 3:13-19.

15. Frede S, Freitag P, Geuting L, et al. Oxygen-regulated expression of the erythropoietin gene in the human renal cell line REPC. Blood. 2011;117:4905-4914.

16. Murayama A, Takeyama K, Kitanaka S, et al. The promoter of the human 25-hydroxyvitamin $\mathrm{D}_{3} 1$ alpha-hydroxylase gene confers positive and negative responsiveness to $\mathrm{PTH}$, calcitonin, and 1 alpha, $25(\mathrm{OH})_{2} \mathrm{D}_{3}$. Biochem Biophys Res Commun. 1998;249:11-16.

17. Takeyama $\mathrm{K}, \mathrm{Kato} \mathrm{S}$. The vitamin $\mathrm{D}_{3}$ 1alpha-hydroxylase gene and its regulation by active vitamin $\mathrm{D}_{3}$. Biosci Biotechnol Biochem. 2011;75:208-213.

18. Kelley R, Werdin ES, Bruce AT, et al. Tubular cell-enriched subpopulation of primary renal cells improves survival and augments kidney function in rodent model of chronic kidney disease. Am J Physiol Renal Physiol. 2010;299:F1026-1039.

19. Hishikawa K, Marumo T, Miura S, et al. Musculin/MyoR is expressed in kidney side population cells and can regulate their function. J Cell Biol. 2005;169:921-928.

20. Osafune $\mathrm{K}$. In vitro regeneration of kidney from pluripotent stem cells. Exp Cell Res. 2010;316:2571-2577.

21. Joraku A, Stern KA, Atala A, et al. In vitro generation of threedimensional renal structures. Methods. 2009;47:129-133.

22. Subramanian B, Rudym D, Cannizzaro C, et al. Tissueengineered three-dimensional in vitro models for normal and diseased kidney. Tissue Eng Part A. 2010;16:2821-2831.

23. Rosines E, Johkura K, Zhang X, et al. Constructing kidneylike tissues from cells based on programs for organ development: toward a method of in vitro tissue engineering of the kidney. Tissue Eng Part A. 2010;16:2441-2455.

24. Haraguchi Y, Shimizu T, Sasagawa T, et al. Fabrication of functional three-dimensional tissues by stacking cell sheets in vitro. Nat Protoc. 2012;7:850-858.

25. Amiel GE, Yoo JJ, Atala A. Renal therapy using tissue-engineered constructs and gene delivery. World J Urol. 2000;18:71-79.

26. Lanza RP, Chung HY, Yoo J, et al. Generation of histocompatible tissues using nuclear transplantation. Nat Biotechnol. 2002;20:689-696.

27. Sekiya S, Shimizu T, Yamato M, et al. Bioengineered cardiac cell sheet grafts have intrinsic angiogenic potential. Biochem Biophys Res Commun. 2006;341:573-582.

28. Sekiya S, Muraoka M, Sasagawa T, et al. Three-dimensional cell-dense constructs containing endothelial cell-networks are an effective tool for in vivo and in vitro vascular biology research. Microvasc Res. 2010;80:549-551.

29. Sasagawa T, Shimizu T, Sekiya $S$, et al. Design of prevascularized three-dimensional cell-dense tissues using a cell sheet stacking manipulation technology. Biomaterials. 2010; 31:1646-1654.

30. Asakawa N, Shimizu T, Tsuda Y, et al. Pre-vascularization of in vitro three-dimensional tissues created by cell sheet engineering. Biomaterials. 2010;31:3903-3909.

Address correspondence to: Okano Teruo, PhD

Institute of Advanced Biomedical Engineering and Science Tokyo Women's Medical University 8-1 Kawada-cho Shinjuku-ku, Tokyo 162-8666 Japan

E-mail: tokano@abmes.twmu.ac.jp 